\title{
Differences in intrapancreatic lipid accumulation between men of White European and Black West African ethnicity with type 2 diabetes.
}

\author{
O. Hakim ${ }^{1}$, C. Mohandas ${ }^{1}$, J.L. Peacock ${ }^{2}$, A.M Umpleby ${ }^{3}$, G. Charles-Edwards ${ }^{4,5}$, S.A. Amiel ${ }^{1}$ \\ and L.M. Goff \\ ${ }^{1}$ Department of Diabetes, School of Life Course Sciences, \\ ${ }^{2}$ School of Population Health and Environmental Sciences, King's College London, SE1 1UL, UK, \\ ${ }^{3}$ Faculty of Health and Medical Sciences, University of Surrey, GU2 7XH, UK, \\ ${ }^{5}$ Medical Physics, Guy's \& St Thomas' NHS Foundation Trust, SE1 7EH, UK and \\ ${ }^{4}$ School of Biomedical Engineering \& Imaging Sciences, King's College London, SE1 7EH, UK
}

Ectopic fat accumulation in the pancreas, called intrapancreatic lipid (IPL), may play a role in the development of type 2 diabetes (T2D) by releasing lipid intermediates that damage beta-cells in a phenomenon termed lipotoxicity (1). People of Black ethnicity are disproportionately affected by T2D despite typically having lower visceral fat and intrahepatic fat compared to their Caucasian counterparts (2), however, there has been limited investigation of IPL in Black populations. Therefore, this study aimed to assess and compare IPL in White European (WE) and Black West African (BWA) men with early T2D.

In a cross-sectional study, $18 \mathrm{WE}$ and 19 BWA men with early T2D underwent magnetic resonance imaging at 1.5-Tesla (Siemens Aera) using a Dixon sequence to produce axial fat and water images from the neck to the knee; MRI data of the abdomen were analysed to determine IPL in the head, body and tail of the pancreas. Within and between each ethnic group, we investigated regional IPL depositions of the head, body and tail regions of the pancreas using a mixed between-within subjects ANOVA.

The WE and BWA men were well matched for age, weight and BMI (all $P>0.05$ ). BWA men exhibited significantly lower mean IPL than WE men (WE: 10.08 (SD 2.46) vs BWA: 8.22 (SD 2.51) \%, $P=0.029$ ), which was driven by a significantly lower IPL in the head of the pancreas in BWA men (WE: 9.66 (SD 3.14) vs BWA: 7.03 (SD 2.65) $\%, P=0.009$ ) (Figure 1). There were significant differences in IPL between the pancreatic regions in BWA men (Wilks' Lambda $=0.791, P=0.019$ ), with a significant main effect for ethnicity $(P=0.029)$ with increasing IPL deposition from the head to the body to the tail of the pancreas (Figure 1). There were no significant differences in the distribution of IPL between the two ethnic groups $(P=0.474)$.

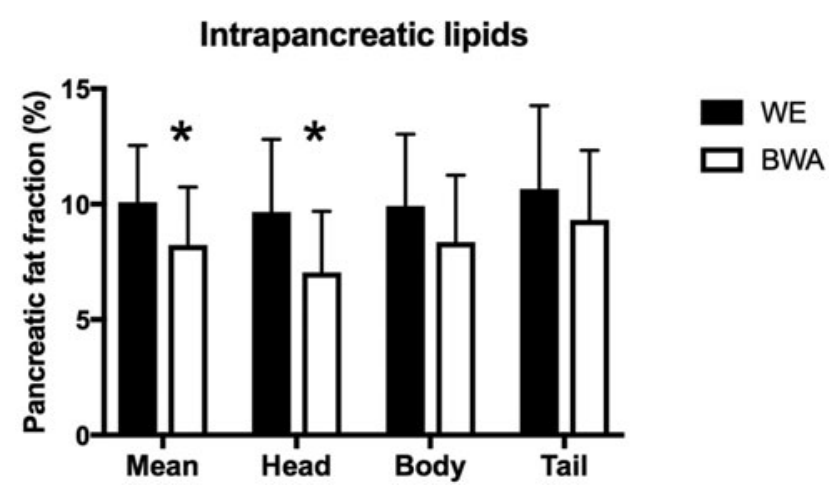

Fig. 1. Mean intrapancreatic lipids and intrapancreatic lipids of the head, body and tail, data presented by ethnicity. Data presented as mean \pm SD * Significant $p<0.05$ as determined by an independent samples t-test between WE and BWA men.

IPL is higher in WE compared to BWA men with early T2D, therefore, we hypothesise that beta-cell lipotoxicity is a more important determinant of the development of T2D in WE compared to BWA men.

1. Al-Mrabeh A, Hollingsworth KG, Steven S et al. (2017) PLoS One 12(4), e0174660

2. Alderete T, Toledo-Corral C \& Goran M (2014) Curr Diab Rep 14(2), 455 